\title{
Molecular Mechanic Studies on the Zwitterionic and Neutral Conformation Stability of Biological Active Peptide
}

\author{
Serda KECEL GUNDUZ ${ }^{*}$, Sefa CELIK ${ }^{2}$ \\ ${ }^{1}$ Istanbul University, Science Faculty, Physics Department, 34134, İstanbul \\ 2Istanbul University, Electrical-Electronics Engineering Department, 34320, İstanbul
}

(Alınış / Received: 13.04.2017, Kabul / Accepted: 14.09.2017, Online Yayınlanma / Published Online: 24.10.2017)

\section{Keywords}

Zwitterionic,

Neutral,

Ala-Gln,

Conformational analysis, DFT

\begin{abstract}
In this current work the conformational properties of the L-Alanyl-LGlutamine dipeptide which was biological active dipeptide has been investigated by theoretical conformational analysis method using program which is based on Molecular Mechanic calculations in order to determine the structure function relation. The calculations on zwitterionic and neutral form of Ala-Gln which is formed side and main chain torsion angles let us to define their preferable energetically conformers. The side and main chain status of the stable conformations of Ala-Gln were acquired based on the results of the conformational analysis. By using Ramachandran maps, the global conformation, which has the lowest energy of the dipeptide, has been determined. The goal of this work was to explain structure-activity relationship by investigating the bioactive conformation of biological active peptide.
\end{abstract}

\section{Biyolojik Aktif Peptidin Zwitterionik ve Nötr Konformasyon Kararlılı̆̆ı üzerine Moleküler Mekanik Araştırmalar}

\section{Anahtar Kelimeler} Zwitteriyonik, Nötral, Ala-Gln, Konformasyon analizi, DFT

\begin{abstract}
Özet: Bu çalışmada, biyolojik aktif dipeptid olan L-Alanil-L-Glutamin dipeptidin konformasyonel özellikleri, yapı fonksiyonu ilişkisini belirlemek için Moleküler Mekanik hesaplamalara dayanan program kullanılarak, teorik konformasyon analizi yöntemi ile araștırılmıștır. Ala-Gln dipeptidinin zwitterionik ve nötr formda yan zincir torsiyon açılarının bir fonksiyonu olarak hesaplanması, enerjik olarak tercih edilen konformasyonlarını belirlememizi sağlar. Elde edilen konformasyonel analiz sonuçlarına bağlı olarak, dipeptide ait stabil konformasyonların yan zincir kalıntılarının göreli pozisyonları elde edilmiștir. Dipeptide ait en düşük enerji konformasyonu Ramachandran haritaları kullanılarak belirlenmiştir. Bu çalışmanın amacı biyolojik aktif dipeptidin biyoaktif konformasyonunu açıklığa kavuşturmak için yapı-aktivite ilişkisini araştırmaktır.
\end{abstract}

\section{Introduction}

Since the electronic and vibrational properties of biologically active peptides are related to its conformational structure, it is very important to know the conformational characteristics of this peptides, to investigate their structural functional properties. Alanyl is a hydrophobic molecule and it can support the body converts glucose to energy, at the same time helps the liver and removes various toxins from the body. Glutamine is necessary for immune system for cell proliferation, it may move through the respiratory tract and increase the function of the stimulated immune cells. Within the muscle, it is important for repair, particularly after trauma. To improve mental function, to control blood sugar levels and to maintain muscle mass, it is also utilized by the body. L-alanyl-L-glutamine (Ala-Gln) dipeptide which is an substantial biological active molecule has various, effective, and important biological activities such as; decreasing effect of infection, dehiscence, abcess, mortality in critically ill patients and length of stay after operation[1], preventive effect of the inhibition of migration, apoptosis[2], improving effect of replenishment of depleted glutathione stores, nitrogen balance, immune defenses, growth performance of piglets[37], increasing effect of protein synthesis, ameliorates immune function[3-5], protective effect of intestinal barrier permeability[3-5], and also shows effective 
properties on control of diabetes in liver transplanted patients, survival and activities of digestive enzyme, enzymatic antioxidative status[89]. It is also used as an immunomodulator in addition to knowing that chemotherapy side effects are diminised.

Besides, the gastrointestinal mucosal protective and antioxidant activities are known. In the gas-phase amino acids, especially exist in the neutral form, however in the water, amino acids are present in the zwitterionic form. A zwitterion form (at neutral $\mathrm{pH}$ values, the amino group (-NH3+) own a positive charge and the carboxyl group (COO-) possess a negative charge) is a structure which has no overall electrical charge, but has apart parts such as positively and negatively charged parts, that exist even in the solid state. These charged moieties cause much stronger ionic forces between the ion and its neighbors or surroundings, rather than weaker hydrogen bonds or other intramolecular forces.

Due to the ionic attractions take more energy to break, melting points of these molecules escalate. In water, the ionic attractions between the ions in the solid amino acid are altered by strong attractions between polar water molecules and the zwitterions. Biological activity is also influenced by the $\mathrm{pH}$ of the water as it determines whether or not certain functional groups are charged, and hence it engenders structural modifications. This makes it possible formation of multiple peptide conformations. Due to this structural modification, the biological functions of these peptides are changed. By determining the spatial structures and the full of low-energy conformational states of biological active peptides allow us to understand the relation between structure- function mechanisms. In present study, the results of conformational analysis of zwitterionic and nutral form of Ala-Gln dipeptide are reported.

\section{Method}

Molecular Mechanic method employs an appropriate algorithm to find the molecular structure of a local energy minimum which correspond to stable conformers of the molecule

Molecular mechanic method with the great computational speed allows us to determine conformational energy and structural searching for peptides. Theoretical conformational analysis method, which is based on the Molecular Mechanics approach, is substantial for conformational compatibility. Calculation of biological active peptide has been performed by the method of theoretical conformational analysis with the help of nonvalent, electrostatic and torsional interactions and energy of the hydrogen bonds. Nonvalent interactions are identified by Lennard-Jones potential with the parameters suggested in [10]. By using Coulombs law which introduces monopole approximation in the partial charges on the atoms in molecule, electrostatic interactions are determined [10]. Hydrogen bonds are described by Morze potential which the value of permittivity is taken to be 10 for zwitterionic surrounding. Amino acid main and side chain dihedral angles are taken from [10] in order to identify torsional potentials and values of rotation barriers. The conformations are performed by the number of rotational degrees of freedom of single bonds of the backbone and side chains for the each amino residue of dipeptide. The rotational angels of each amino acide residue is characterized by the backbone $\varphi, \psi$ and side chain $\chi_{1}, \chi_{2} \ldots$ dihedral angles. The $\varphi$ and $\psi$ dihedral angles are located in lowenergy region $R\left(\varphi, \psi=\left(-180^{\circ}-0^{\circ}\right), B\left(\varphi=\left(-180^{\circ}\right)-0^{\circ}\right.\right.$; $\left.\psi=0^{\circ}-180^{\circ}\right), \quad \mathrm{L}\left(\varphi, \psi=0^{\circ}-180^{\circ}\right) \quad$ and $\mathrm{P}\left(\varphi=0^{\circ}-180^{\circ}\right.$; $\left.\psi=\left(-180^{\circ}\right)-0^{\circ}\right)$ of the conformational map.

Experimental parameters for bond lengths, angles and dihedrals are taken as suggested by Corey and Pauling [11]. The spatial structures and the full complement of low-energy conformational states of the dipeptides was carried out by using program proposed by Godjayev et al.[12]. This study is a review of the structural and energy shift in comparison with zwitterionic and neutral form of biologically active peptid molecule.

\section{Results and Discussion}

\subsection{Structure of zwiterrionic form}

The initial conformations of the Ala-Gln peptide were formed by combining low-energy structures of each amino acide residues. $\mathrm{B}, \mathrm{R}$ and $\mathrm{L}$ areas for Alanin and Glutamine were defined and the values of $\varphi$ and $\psi$ dihedral angles were taken from these regions. Side chains dihedral angles values for Alanin was teken $60^{\circ}, 180^{\circ},-60^{\circ}$ for $\chi_{1}$ and for Glutamine was teken $60^{\circ}, 180^{\circ},-60^{\circ}$ for $\chi_{1}$ and $\chi_{2}, 90^{\circ},-90^{\circ}$ for $\chi_{3}$. 1134 conformers for Ala-Gln dipeptide were investigated for zwitterionic form, one by one. The most stable conformation of zwitterionic forms are in $\mathrm{L}_{3} \mathrm{~B}_{131}$ region and extended (e) shape on Ramachandran maps.

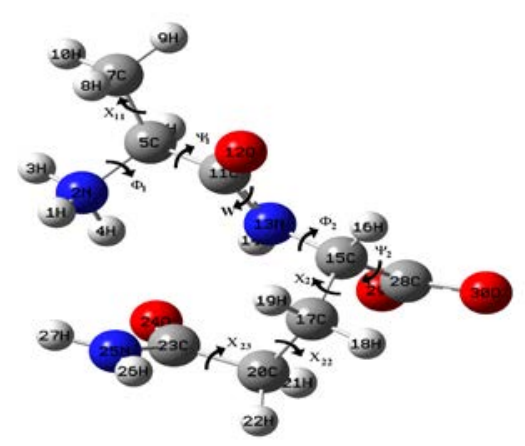

Figure 1. The global conformation of the zwitterionic form of the Ala-Gln dipeptide obtained with LB region. 


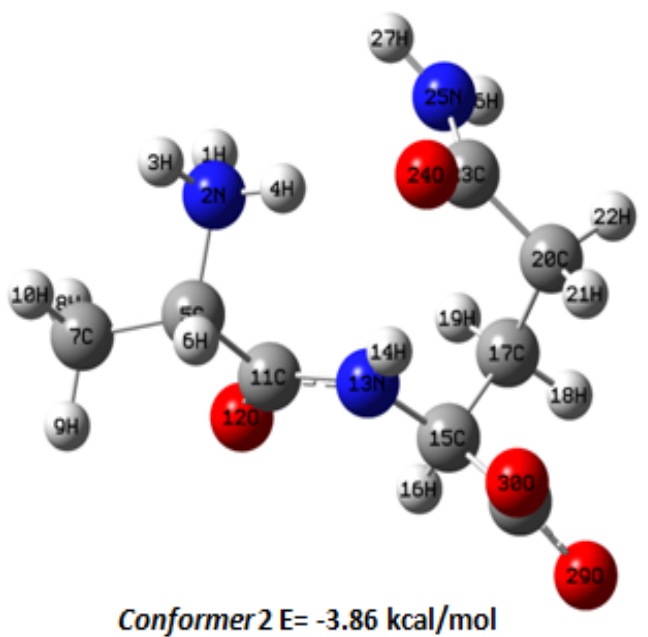

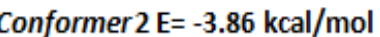

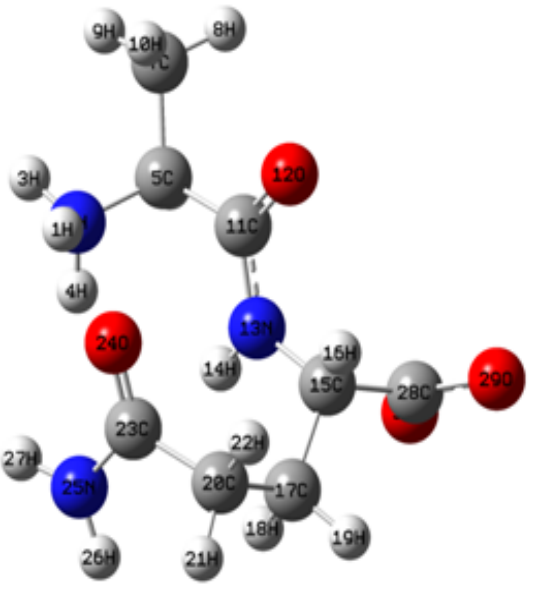

Conformer $4 \mathrm{E}=-3.25 \mathrm{kcal} / \mathrm{mol}$

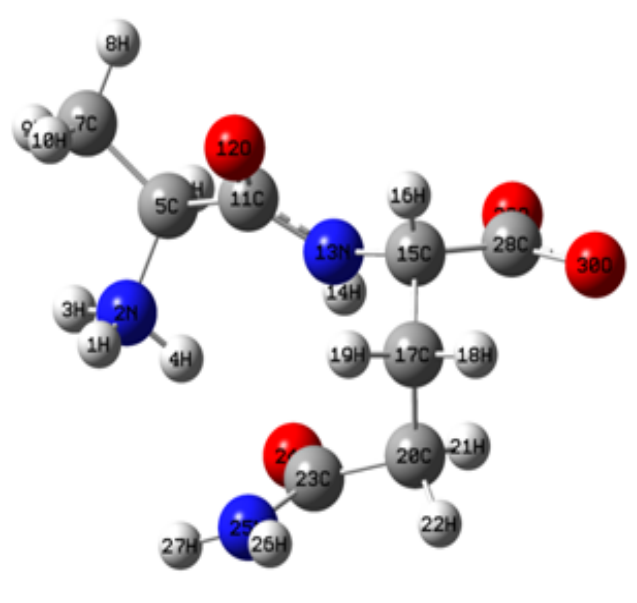

Conformer $3 \mathrm{E}=-3.53 \mathrm{kcal} / \mathrm{mol}$

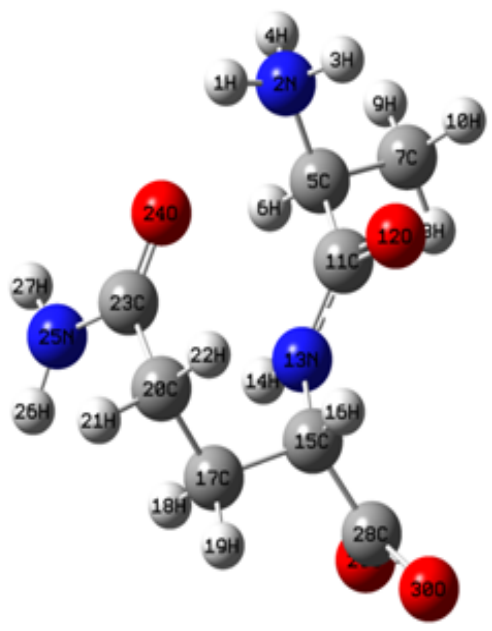

Conformer $5 \mathrm{E}=-3.15 \mathrm{kcal} / \mathrm{mol}$

Figure 2. The four stable conformers of zwitterionic form of Ala-Gln dipeptide in 1134 conformers.

Table 1. The dihedral angle values obtained for the zwitterionic form of the Ala-Gln molecule.

\begin{tabular}{|c|c|c|c|c|c|c|c|c|c|c|c|}
\hline & $\begin{array}{c}\text { Energy } \\
\text { kcal/mol }\end{array}$ & & PHI1 & CH11 & PSI1 & W2 & PHI2 & CH21 & CH22 & CH23 & PSI2 \\
\hline & Exp. & & $58.6^{a}$ & $55.9^{a}$ & $103.67^{a}$ & & $\begin{array}{c}162.9 \text { b,xray } \\
164 \text { b,neu }\end{array}$ & $\begin{array}{c}65.7 \text { b,xray } \\
66.15 \text { b,neu }\end{array}$ & $\begin{array}{l}175.5^{\text {b,xray }} \\
175.5^{\text {b,neu }}\end{array}$ & $\begin{array}{l}167.1 \text { b,xray } \\
167.2^{\text {b,neu }} \\
\end{array}$ & $\begin{array}{c}167.2^{\text {b,xray }} \\
167 \text { b,neu }\end{array}$ \\
\hline \multirow{2}{*}{1} & \multirow{2}{*}{-4.19} & IN & 50.000 & 60.000 & 60.000 & 180.000 & -150.000 & 60.000 & -60.000 & 90.000 & 150.000 \\
\hline & & OUT & 56.874 & 58.332 & 72.521 & 180.708 & -150.636 & 58.468 & -74.807 & 102.292 & 158.172 \\
\hline \multirow{2}{*}{2} & \multirow{2}{*}{-3.86} & IN & 50.000 & 60.000 & 60.000 & 180.000 & -100.000 & 60.000 & -60.000 & 90.000 & -60.000 \\
\hline & & OUT & 56.462 & 58.026 & 73.122 & 181.236 & -142.786 & 56.333 & -77.555 & 101.143 & -14.082 \\
\hline \multirow[b]{2}{*}{3} & \multirow{2}{*}{-3.53} & IN & 50.000 & 180.000 & 60.000 & 180.000 & -100.000 & 60.000 & -60.000 & 90.000 & 140.000 \\
\hline & & OUT & 55.532 & 177.827 & 72.875 & 181.193 & -141.336 & 57.582 & -78.330 & 101.154 & 193.492 \\
\hline \multirow{2}{*}{4} & \multirow{2}{*}{-3.25} & IN & 50.000 & 180.000 & 60.000 & 180.000 & -90.000 & -60.000 & 60.000 & -90.000 & -40.000 \\
\hline & & OUT & 47.485 & 178.133 & 65.707 & 182.911 & -97.420 & -70.857 & 65.541 & -108.085 & -42.634 \\
\hline \multirow{2}{*}{5} & \multirow{2}{*}{-3.15} & IN & -115.000 & 180.000 & 140.000 & 180.000 & -150.000 & -60.000 & 60.000 & -90.000 & 150.000 \\
\hline & & OUT & -61.156 & 178.409 & 129.447 & 184.417 & -114.909 & -65.381 & 70.432 & -114.725 & 146.992 \\
\hline \multirow{2}{*}{6} & \multirow{2}{*}{-3.05} & IN & -115.000 & 180.000 & 140.000 & 180.000 & -90.000 & -60.000 & 60.000 & -90.000 & -40.000 \\
\hline & & OUT & -55.901 & 180.218 & 139.178 & 183.263 & -111.407 & -67.759 & 68.718 & -114.666 & -50.091 \\
\hline \multirow{2}{*}{7} & \multirow{2}{*}{-2.85} & IN & -115.000 & 180.000 & 140.000 & 180.000 & -100.000 & -60.000 & 60.000 & -90.000 & 140.000 \\
\hline & & OUT & -60.351 & 178.556 & 140.600 & 182.613 & -110.561 & -68.381 & 68.942 & -114.573 & 139.268 \\
\hline \multirow{2}{*}{8} & \multirow{2}{*}{-2.83} & IN & -115.000 & 60.000 & 140.000 & 180.000 & -100.000 & -60.000 & 60.000 & -90.000 & -60.000 \\
\hline & & OUT & -66.795 & 57.559 & 143.351 & 182.835 & -108.871 & -67.242 & 67.388 & -113.261 & -58.166 \\
\hline \multirow{2}{*}{9} & \multirow{2}{*}{-2.80} & IN & -90.000 & 60.000 & -50.000 & 180.000 & -90.000 & 60.000 & -60.000 & 90.000 & -40.000 \\
\hline & & OUT & -44.621 & 58.646 & -57.608 & 178.340 & -146.040 & 67.626 & -67.613 & 106.194 & -16.729 \\
\hline \multirow{2}{*}{10} & \multirow{2}{*}{-2.74} & IN & -90.000 & 180.000 & -50.000 & 180.000 & -100.000 & 60.000 & -60.000 & 90.000 & 140.000 \\
\hline & & OUT & -54.214 & 173.103 & -64.600 & 178.222 & -141.799 & 67.720 & -69.290 & 105.841 & 165.607 \\
\hline
\end{tabular}


Table 2. The total energy calculated for the zwitterionic Ala-Gln molecule and the contribution terms that make up this energy.

\begin{tabular}{|c|c|c|c|c|c|}
\hline & $\begin{array}{c}\text { Total } \\
\text { Energy } \\
\text { (kcal/mol) }\end{array}$ & $\frac{\searrow}{\frac{\pi}{\pi}}$ & $\begin{array}{c}E_{\text {van der Walls }} \\
\text { (kcal/mol) }\end{array}$ & $\begin{array}{l}\text { E electrostatic } \\
\text { (kcal/mol) }\end{array}$ & $\begin{array}{c}\text { Etorsion }_{\text {tol }} \\
\text { (kcal/mol) }\end{array}$ \\
\hline 1 LB1 & -4.19 & $\mathbf{e}$ & -6.77 & 1.15 & 1.43 \\
\hline 2 LR2 & -3.86 & $\mathrm{e}$ & -6.56 & 1.08 & 1.62 \\
\hline 3 LB2 & -3.53 & $\mathrm{e}$ & -6.17 & 1.17 & 1.48 \\
\hline 4 LR1 & -3.25 & e & -5.83 & 1.46 & 1.12 \\
\hline 5 BB1 & -3.15 & $\mathrm{e}$ & -5.27 & 0.81 & 1.31 \\
\hline 6 BR1 & -3.05 & $\mathrm{e}$ & -5.18 & 0.99 & 1.13 \\
\hline 7 BB2 & -2.85 & $\mathrm{e}$ & -5.14 & 1.01 & 1.28 \\
\hline 8 BR2 & -2.83 & $\mathrm{e}$ & -4.89 & 1.01 & 1.04 \\
\hline 9 RR1 & -2.80 & $\mathrm{f}$ & -5.46 & 1.49 & 1.17 \\
\hline 10 RB2 & -2.74 & $\mathrm{f}$ & -5.40 & 1.46 & 1.20 \\
\hline
\end{tabular}

The most stable structure for zwitterionic form is obtained by $\chi 11=60^{\circ}, \chi 21=60^{\circ}, \chi 22=-60^{\circ}$ and $\chi 23=90^{\circ}$ is given in Figure 1. Dihedral angles of the ten possible stable conformations before and after energy optimization and relative total energies are showed in Table 1. Van der Waals, electrostatic, torsional energies which generate total energy for ten conformers are given Table 2 . The possible four conformers which have low energy are also demonstrated in Figure 2.

\subsection{Structure of neutral form}

1134 conformers for Ala-Gln dipeptide were investigated for neutral form, one by one. The most stable conformation of neutral form are determined in LR1 region and extended (e) shape on Ramachandran maps.

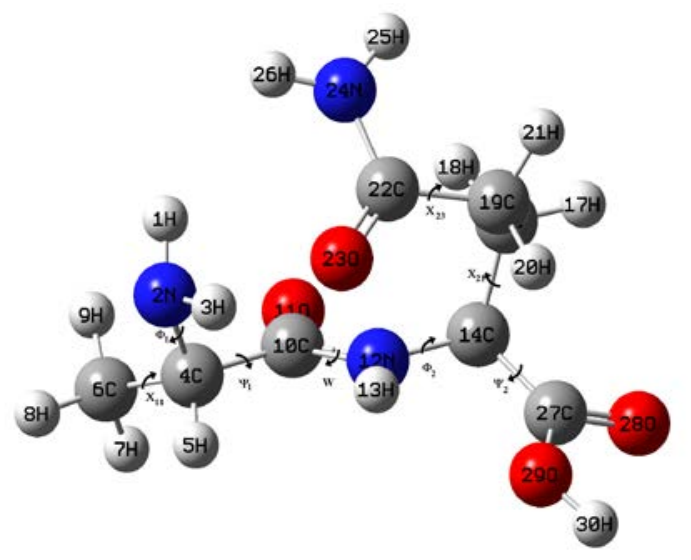

Figure 3. The global conformation of the neutral form of Ala-Gln dipeptide with the atom numbering in LR1 region.

The most stable structure for neutral form is obtained by $\chi_{11}=180^{\circ}, \chi_{21}=60^{\circ}, \chi_{22}=-60^{\circ}$ and $\chi_{23}=90^{\circ}$. is given in Figure 3. Dihedral angles of the ten possible stable conformations before and after energy optimization and relative total energies are showed in Table 3. The total energy with the sum of Van der Waals, electrostatic, torsional energies for ten conformers are given in Table 4. The possible four conformers which have low energy are also demonstrated in Figure 4.

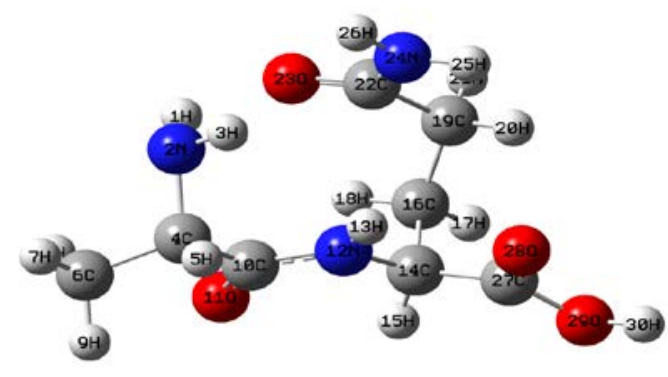

Conformer $2 \mathrm{E}=-\mathbf{0 . 1 8} \mathrm{kcal} / \mathrm{mol}$

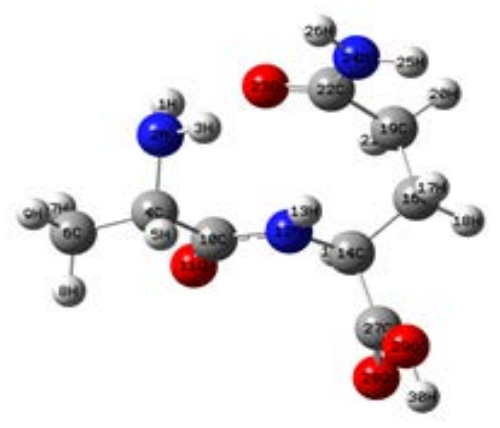

Conformer $4 \mathrm{E}=0.14 \mathrm{kcal} / \mathrm{mol}$

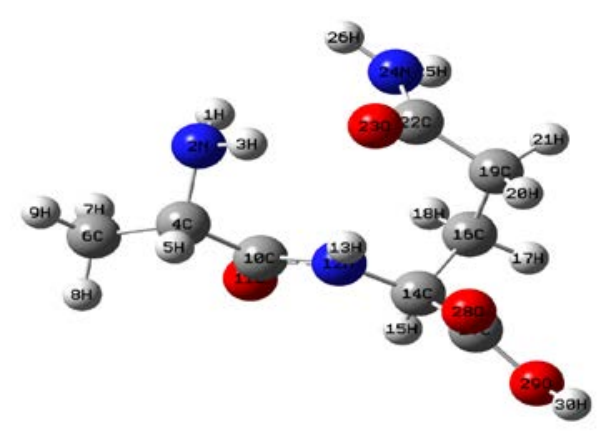

Conformer $3 \mathrm{E}=0.04 \mathrm{kcal} / \mathrm{mol}$

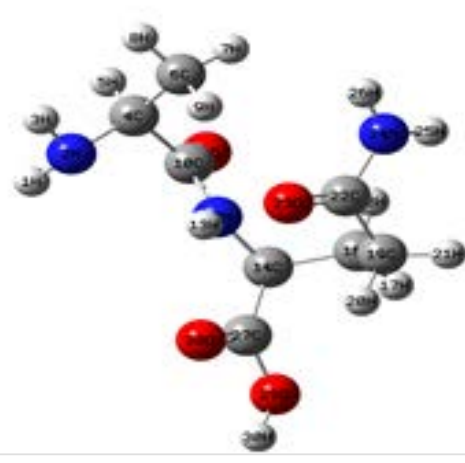

Conformer $5 \mathrm{E}=1 \mathrm{kcal} / \mathrm{mol}$

Figure 4. The four more stable conformation of the neutral form of Ala-Gln dipeptide with their energy. 
Table 3. The dihedral angle values obtained for the neutral form of the Ala-Gln molecule.

\begin{tabular}{|c|c|c|c|c|c|c|c|c|c|c|c|}
\hline & $\begin{array}{c}\begin{array}{c}\text { Energy } \\
\text { kcal/mol }\end{array} \\
\end{array}$ & & PHI1 & CH11 & PSI1 & $W 2$ & PHI2 & $\mathrm{CH} 21$ & $\mathrm{CH} 22$ & $\mathrm{CH} 23$ & PSI2 \\
\hline & Exp. & & $58.6^{a}$ & $55.9^{a}$ & $103.67^{a}$ & & $\begin{array}{c}162.9 \mathrm{~b}, \text { xray } \\
164 \text { b,neu }\end{array}$ & $\begin{array}{l}65.7 \text { b,xray } \\
66.15 \text { b,neu }\end{array}$ & $\begin{array}{l}175.5^{\text {b,xray }} \\
175.5 \text { b,neu }\end{array}$ & $\begin{array}{l}167.1 \text { b,xray } \\
167.2^{\text {b,neu }}\end{array}$ & $\begin{array}{c}167.2 \text { b,xray } \\
167 \text { b,neu }\end{array}$ \\
\hline \multirow{2}{*}{1} & \multirow{2}{*}{-0.30} & IN & 50.000 & 180.000 & 60.000 & 180.000 & -90.000 & 60.000 & -60.000 & 90.000 & -40.000 \\
\hline & & OUT & 49.409 & 179.261 & 75.708 & 180.713 & -145.769 & 55.251 & -76.967 & 104.510 & -18.820 \\
\hline \multirow{2}{*}{2} & \multirow{2}{*}{-0.18} & IN & 50.000 & 180.000 & 60.000 & 180.000 & -150.000 & 60.000 & -60.000 & 90.000 & 150.000 \\
\hline & & OUT & 50.275 & 179.713 & 71.746 & 180.870 & -150.316 & 58.251 & -76.214 & 101.434 & 156.782 \\
\hline \multirow{2}{*}{3} & \multirow{2}{*}{0.04} & IN & 50.000 & -60.000 & 60.000 & 180.000 & -100.000 & -60.000 & 180.000 & -90.000 & 140.000 \\
\hline & & OUT & 33.546 & -60.754 & 72.750 & 182.115 & -158.090 & -61.953 & 178.574 & -104.179 & 134.931 \\
\hline \multirow{2}{*}{4} & \multirow{2}{*}{0.14} & IN & 50.000 & 180.000 & 60.000 & 180.000 & -100.000 & 180.000 & 60.000 & -90.000 & -60.000 \\
\hline & & OUT & 43.119 & 179.003 & 81.467 & 179.776 & -104.962 & 181.206 & 63.119 & -103.098 & -61.689 \\
\hline \multirow{2}{*}{5} & \multirow{2}{*}{1.00} & IN & -90.000 & 180.000 & -50.000 & 180.000 & -100.000 & 60.000 & -60.000 & 90.000 & 140.000 \\
\hline & & OUT & -60.953 & 175.387 & -49.048 & 178.043 & -144.085 & 65.390 & -71.154 & 106.662 & 171.051 \\
\hline \multirow{2}{*}{6} & \multirow{2}{*}{1.05} & IN & -90.000 & -60.000 & -50.000 & 180.000 & -100.000 & 60.000 & -60.000 & 90.000 & -60.000 \\
\hline & & OUT & -64.941 & -66.141 & -49.923 & 178.843 & -146.962 & 66.835 & -68.998 & 108.171 & -5.951 \\
\hline \multirow{2}{*}{7} & \multirow{2}{*}{1.15} & IN & -115.000 & 180.000 & 140.000 & 180.000 & -100.000 & -60.000 & -60.000 & 90.000 & -60.000 \\
\hline & & OUT & -173.569 & 181.382 & 140.187 & 182.105 & -105.842 & -54.222 & -59.392 & 109.009 & -56.236 \\
\hline \multirow{2}{*}{8} & \multirow{2}{*}{1.16} & IN & -90.000 & -60.000 & -50.000 & 180.000 & -150.000 & -60.000 & -60.000 & 90.000 & 150.000 \\
\hline & & OUT & -47.417 & -61.987 & -57.606 & 182.820 & -105.073 & -52.647 & -58.335 & 108.879 & 145.591 \\
\hline \multirow{2}{*}{9} & \multirow{2}{*}{1.19} & IN & -90.000 & 180.000 & -50.000 & 180.000 & -90.000 & -60.000 & -60.000 & -90.000 & -40.000 \\
\hline & & OUT & -45.628 & 177.701 & -57.620 & 179.613 & -117.776 & -65.404 & -71.739 & -80.926 & -45.310 \\
\hline \multirow{2}{*}{10} & \multirow{2}{*}{1.20} & IN & -115.000 & 180.000 & 140.000 & 180.000 & -90.000 & -60.000 & 60.000 & 90.000 & -40.000 \\
\hline & & OUT & -172.000 & 181.492 & 141.077 & 180.729 & -107.307 & -70.041 & 66.530 & 60.204 & -48.035 \\
\hline
\end{tabular}

a: Ref [15]; b: Ref [16]

Table 4. The total energy calculated for the zwitterionic Ala-Gln molecule and the contribution terms that make up this energy.

\begin{tabular}{|c|c|c|c|c|c|}
\hline & $\begin{array}{c}\text { Total Energy } \\
\text { kcal/mol }\end{array}$ & 产 & $\begin{array}{l}\mathbf{E}_{\text {van der Walls }} \\
\mathbf{k c a l} / \mathbf{m o l} \\
\end{array}$ & $\begin{array}{l}\mathbf{E}_{\text {electrostatic }} \\
\text { kcal/mol } \\
\end{array}$ & $\begin{array}{c}\begin{array}{c}E_{\text {torsion }} \\
\mathrm{kcal} / \mathrm{mol}\end{array} \\
\end{array}$ \\
\hline 1 LR1 & -0.30 & $\mathbf{e}$ & -6.53 & 4.65 & 1.58 \\
\hline 2 LB1 & -0.18 & $\mathrm{e}$ & -6.06 & 4.41 & 1.47 \\
\hline 3 LB2 & 0.04 & $\mathrm{e}$ & -5.50 & 4.41 & 1.12 \\
\hline 4 LR2 & 0.14 & $\mathrm{e}$ & -6.22 & 5.32 & 1.04 \\
\hline 5 RB2 & 1.00 & $\mathrm{f}$ & -5.52 & 5.31 & 1.21 \\
\hline 6 RR2 & 1.05 & $\mathrm{f}$ & -5.96 & 5.62 & 1.39 \\
\hline 7 BR2 & 1.15 & $\mathrm{e}$ & -5.27 & 5.51 & 0.91 \\
\hline 8 RB1 & 1.16 & $\mathrm{f}$ & -5.66 & 5.90 & 0.92 \\
\hline 9 RR1 & 1.19 & $\mathrm{f}$ & -6.13 & 6.42 & 0.90 \\
\hline $10 \mathrm{BR} 1$ & 1.20 & $\mathrm{e}$ & -5.51 & 5.60 & 1.10 \\
\hline
\end{tabular}

Table 5. Hydrogen bonds and hydrogen bonding energies for zwitterionic form

\begin{tabular}{ccc}
\hline Atoms & $\begin{array}{c}\text { Hydrogen Bond } \\
\circ\end{array}$ & $\begin{array}{c}\text { Hydrogen } \\
\text { bonding energy } \\
\text { (kcal/mol) }\end{array}$ \\
\hline $4-24$ & 1,92 & $-1,36$ \\
$14-24$ & 2,24 & $-0,70$ \\
$14-29$ & 2,32 & $-0,56$ \\
\hline
\end{tabular}

Table 6. Interaction energies $(\mathrm{kcal} / \mathrm{mol})$ between side and main chains for zwitterionic form.

\begin{tabular}{ccccc}
$\begin{array}{c}\text { Ala- } \\
\text { main }\end{array}$ & Ala-side & $\begin{array}{c}\text { Gln- } \\
\text { main }\end{array}$ & \multicolumn{1}{c}{$\begin{array}{c}\text { Gln- } \\
\text { side }\end{array}$} & \multicolumn{1}{c}{ Ala- } \\
\cline { 1 - 4 } 2,44 & 0,60 & $-3,34$ & $-4,39$ & $\begin{array}{c}\text { main } \\
\text { Ala-side } \\
\end{array}$ \\
0 & $-0,32$ & $-0,15$ & $\begin{array}{c}\text { Gln- } \\
\text { main } \\
\text { Gln- } \\
\text { side }\end{array}$ \\
\hline
\end{tabular}

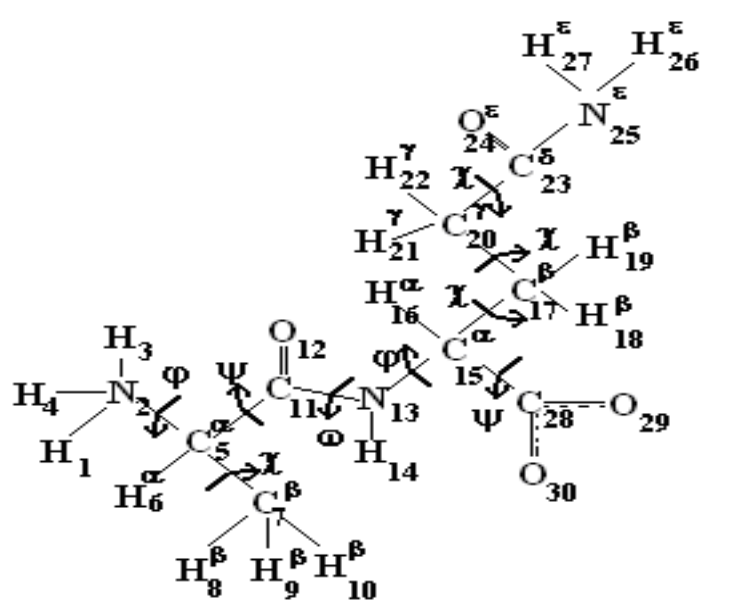

Figure 5. The most stable conformation of the zwitterionic form of Ala-Gln dipeptide.

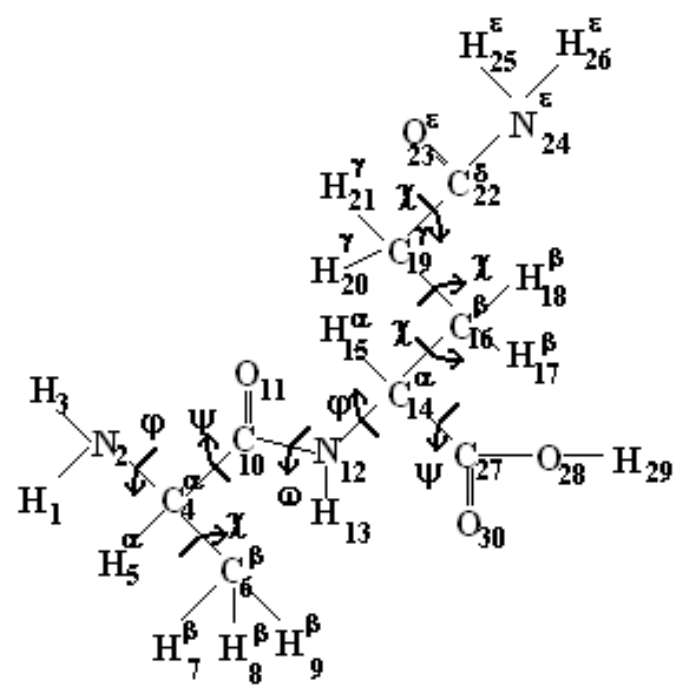

Figure 6. The most stable conformation of the neutral form of Ala-Gln dipeptide. 
Table 7. Hydrogen bonds and hydrogen bonding energies for neutral form

\begin{tabular}{ccc}
\hline Atoms & $\begin{array}{c}\text { Hydrogen Bond } \\
\circ\end{array}$ & $\begin{array}{c}\text { Hydrogen } \\
\text { bonding energy } \\
\text { (kcal/mol) }\end{array}$ \\
\hline $3-23$ & $1,9)$ & $-0,95$ \\
$13-23$ & 2,25 & $-0,49$ \\
$13-28$ & 2,29 & $-0,45$ \\
\hline
\end{tabular}

Table 8. Interaction energies $(\mathrm{kcal} / \mathrm{mol})$ between side and main chains for neutral form.

\begin{tabular}{|c|c|c|c|c|}
\hline $\begin{array}{c}\text { Ala- } \\
\text { main }\end{array}$ & Ala-side & $\begin{array}{c}\text { Gln- } \\
\text { main }\end{array}$ & $\begin{array}{l}\text { Gln- } \\
\text { side }\end{array}$ & \\
\hline \multirow[t]{3}{*}{4,76} & 0,81 & $-2,95$ & $-4,68$ & $\begin{array}{l}\text { Ala- } \\
\text { main }\end{array}$ \\
\hline & 0 & $-0,29$ & $-0,15$ & Ala-side \\
\hline & & 4,65 & $\begin{array}{l}-0,28 \\
-1,79\end{array}$ & $\begin{array}{c}\text { GIn- } \\
\text { main } \\
\text { Gln- } \\
\text { side }\end{array}$ \\
\hline
\end{tabular}

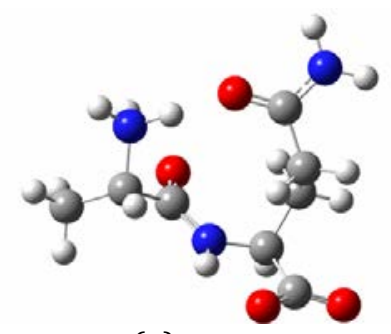

(a)

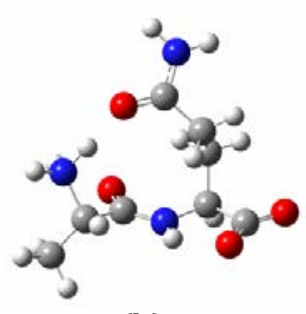

(b)
Figure 7. Comparison of theoretical conformational analysis (a) and DFT/B3LYP 6-31++G(d,p) (b) optimized results of zwitterionic form.

Table 9. Calculated energies (kcal/mol) of Zwitterionic form of Ala-Gln

\begin{tabular}{ccc}
$\begin{array}{c}\text { Zwitterionic } \\
\text { Ala-Gln }\end{array}$ & $\begin{array}{c}\text { DFT-RB3LYP } \\
\text { 6-31G++(d,p) }\end{array}$ & DFT-RB3LYP \\
\hline \multirow{2}{*}{ Energy } & $-779.11536503 \mathrm{Giu}$ & $-779.07174977 \mathrm{au}$ \\
& $-488894,89 \mathrm{kcal} / \mathrm{mol}$ & $-488867,5229 \mathrm{kcal} / \mathrm{mol}$ \\
\hline
\end{tabular}

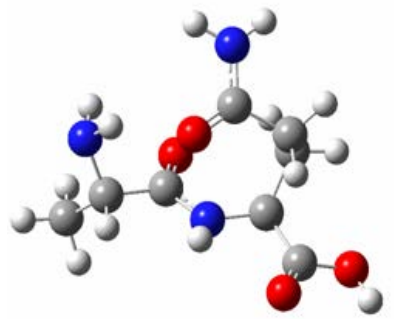

(a)

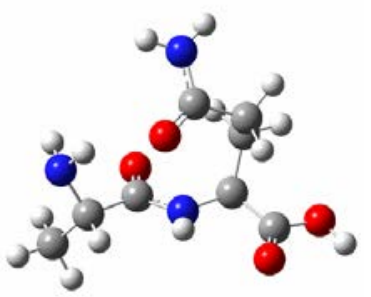

(b)
Figure 8. Comparison of theoretical conformational analysis (a) and DFT/B3LYP 6-31++G(d,p) (b) optimized results of neutral form.

Table 9. Calculated energies (kcal/mol) of Neutral form of Ala-Gln

\begin{tabular}{ccc} 
Ala-Gln & & \\
\hline Nötral & DFT-RB3LYP & DFT-RB3LYP \\
Ala-Gln & $\mathbf{6 - 3 1 G + + ( d , p ) ~}$ & $\mathbf{6 - 3 1 G ( d , p )}$ \\
Energy & $-779.15006391 \mathrm{au}$ & $-779.11134355 \mathrm{au}$ \\
& $-488916.6651 \mathrm{kcal} / \mathrm{mol}$ & $-488892.368 \mathrm{kcal} / \mathrm{mol}$ \\
\hline
\end{tabular}

\section{Conclusion}

The calculated energy, which was performed theoretically conformational analysis method, for zwitterionic form in 1134 conformational region is given (Etot $=-4.19 \mathrm{kcal} / \mathrm{mol}$ ) as the sum of the EvdW $=-6.77 \mathrm{kcal} / \mathrm{mol}$, Eel $=1.15 \mathrm{kcal} / \mathrm{mol}$ and Etor $=1.43$ $\mathrm{kcal} / \mathrm{mol}$ energies. We have determined optimized values of the dihedral angles of backbone and side chains of zwitterionic Ala-Gln dipeptide; its values are $\varphi 1=56.87, \quad c 11=58.33, \quad \Psi 1=72.52 \mathrm{~W}=180.70$, $\varphi 2=-150.63, \quad c 21=58.46, \quad c 22=-74.80, \quad$ c23 $=102.29$, $\Psi 2=158.17$. The most stable conformation is in LB1 region with the (e) shape on Ramachandran maps. The total energy of global conformation of neutral Ala-Gln molecule which was calculated by [12] (Etot $=-0.30 \mathrm{kcal} / \mathrm{mol}$ ) is given as the sum of the van der Waals $(\mathrm{EvdW}=-6.53 \mathrm{kcal} / \mathrm{mol})$, electrostatic $(\mathrm{Eel}=$ $4.65 \mathrm{kcal} / \mathrm{mol}$ ), and torsional interactions (Etor $=1.58$ $\mathrm{kcal} / \mathrm{mol}$ ) energies. The optimized values of the dihedral angles of backbone and side chains of neutral the Ala-Gln dipeptide are found to be; , $\varphi 1=49.40, \mathrm{c} 11=179.26, \Psi 1=75.70 \mathrm{~W}=180.71, \varphi 2=-$ 145.76, $\quad$ c21 $=57.25, \quad$ c22 $=-76.96, \quad$ c23 $=104.51$, $\Psi 2=18.82$ In 1134 conformers for neutral Ala-Gln molecule the most stable conformation is in LR1 region with extended (e) shape on Ramachandran maps. The experimental dihedral values for alanine and glutamine amino acides were also added in Table 1 and 3 . When the theoretical and experimental values are compared, the zwitterionic form is more compatible with the experimental values in Table 1.

When we compare zwitterionic and neutral forms of dipeptide, we obtained that the remarkable contribution on global conformation was Van der Waals interaction with $-6.77 \mathrm{kcal} / \mathrm{mol}$ and -6.53 $\mathrm{kcal} / \mathrm{mol}$ for zwiterionic and neutral form, respectively. The electrostatic and torsion interaction provide detrimental effect on global conformation. The calculated values of electrostatic interaction are $1.15 \mathrm{kcal} / \mathrm{mol}$ and $4.65 \mathrm{kcal} / \mathrm{mol}$ for zwiterionic and neutral form, respectively. This detrimental effect was observed more in the neutral form. While a hydrogen bond between the hydrogen and Oksigen atoms (H4-024) gives rise to global conformation with the contribition of $-1,36 \mathrm{kcal} / \mathrm{mol}$ for zwitterionic form, in neutral form this hydrogen bond (H3-023)has a little weak contribution of global conformation of $-0,95 \mathrm{kcal} / \mathrm{mol}$, see in Table 5 . and Table 7.When we analyze the interaction energies $(\mathrm{kcal} / \mathrm{mol})$ between side and main chains for zwitterionic form, we concluded that Glutamine side chain and Alanin main chain interaction ensures stable conformation of the dipeptide with $4,39 \mathrm{kcal} / \mathrm{mol}$ energy in Table 6 . This interaction was obtained a little bit higher than zwitterionic form with $-4,68 \mathrm{kcal} / \mathrm{mol}$. in Table 8 . The main chain dihedral angles of conformer2 and conformer3 for zwitterionic and neutral Ala-Gln determined as 2 ( $\varphi 1=50 \mathrm{o}, \psi 1=60 \mathrm{o}, w 2=180 \mathrm{o}, \varphi 2=-100 \mathrm{o}, \psi 2=-60 \mathrm{o})$, $3(\varphi 1=50 \mathrm{o}, \psi 1=60 \mathrm{o}, \mathrm{w} 2=180 \mathrm{o}, \varphi 2=-100 \mathrm{o}, \psi 2=140 \mathrm{o})$ and $2(\varphi 1=50 \mathrm{o}, \quad \psi 1=60 \mathrm{o}, \quad w 2=180 \mathrm{o}, \varphi 2=-150 \mathrm{o}$, $\psi 2=150 \mathrm{o}), 3(\varphi 1=50 \mathrm{o}, \psi 1=60 \mathrm{o}, \mathrm{w} 2=180 \mathrm{o}, \varphi 2=-100 \mathrm{o}$, $\psi 2=140 \mathrm{o}$ ) have an total energy $-3.86,-3.53$ and - 
$0.18,0.04 \mathrm{kcal} / \mathrm{mol}$, respectively in Table 1 and Table 3.

The result of theoretical conformation analysis programs were used as starting geometry for quantum chemical calculations. [13-14]. Determination of conformational probabilities of biological macromolecules of zwiterrionic and neutral form of Ala-Gln dipeptide is very considerable to grasp their functions of a drug. The more effective structural analogues may be synthesis based on this conformational analysis results. The conformational energy and structure shifts are found to be in good qualitative agreement for zwitterionic and neutral form of Ala-Gln-dipeptide.

\section{References}

[1] Oguz, M., Kerem, M., Bedirli, A., Mentes, B. B., Sakrak, O., Salman, B., \& Bostanci, H. 2007. l-Alanin-l-glutamine supplementation improves the outcome after colorectal surgery for cancer. Colorectal Disease, 9(6), 515-520.

[2] Brito, G. A., Carneiro-filho, B., Oriá, R. B., Destura, R. V., Lima, A. A., \& Guerrant, R. L. 2005. Clostridium difficile toxin $\mathrm{A}$ induces intestinal epithelial cell apoptosis and damage: role of Gln and Ala-Gln in toxin A effects. Digestive diseases and sciences, 50(7), 1271-1278.

[3] Cynober L. 1997. Glutamine as an activator of immunecells: how does it work? Nutrition,13:688-9.

[4] Morlion B.J., Stehle P., Wachtler P., Siedhoff HP , Köller M. , König W. , Fürst P, Puchstein C. 1998. Total parenteral nutrition with glutamine dipeptide after majör abdominal surgery: a randomized, double-blind, controlled study. Ann Surg;227:302-8.

[5] Mertes N, Schulzki C., Goeters C., Winde, G., Benzing, S., Kuhn, K. S. 2000. Cost containment through L-alanyl-L-glutamine supplemented total parenteral nutrition after major abdominal surgery: a prospective randomized double-blind controlled study. ClinNutr; 19: 395-401.

[6] Zhang, B., Lin, M., Yu, C., Li, J., Zhang, L., Zhou, P., Zhou,G. 2016 Alanyl-glutamine supplementation regulates mTOR and ubiquitin proteasome proteolysis signaling pathways in piglets. Nutrition, 32(10), 1123-1131.

[7] Ockenga, J., Borchert, K., Rifai, K. 2002. Effect of glutamine-enriched total parenteral nutrition in patients with acute pancreatitis Clin Nutr, 21: 409-416

[8] Korraa, A. E. D. A., Labib, H. A., Salah, D. 2016. Studying the effect of parenterally administered l-alanyl l-glutamine dipeptide in diabetes and new onset diabetes in liver transplantation. Egyptian Journal of Anaesthesia, 32(3), 415420.
[9] Chen, X. M., Guo, G. L., Sun, L., Yang, Q. S., Wang, G. Q., Qin, G. X., \& Zhang, D. M. 2016. Effects of Ala-Gln feeding strategies on growth, metabolism, and crowding stress resistance of juvenile Cyprinus carpio var. Jian. Fish \& shellfish immunology, 51, 365-372.

[10] Momany F.A., McGuire R.F., Burgess A.W., Scheraga H.A. 1975. "Energy parameters in polypeptides. VII. Geometric parameters, partial atomic charges, nonboded interactions, hydrogen bond interaction and intrinsictorsional potentials for naturally occurring aminoacid", J. Phys. Chem., vol. 29, 2361-2381.

[11] Popov, E. M., Godjaev, N. M., Ismailova, L. I., Musaev, S. M., Aliev, R. E., Akhmedov, N. A., \& Maksumov, I. S. 1982. A-Priori calculation of spatial structure of bovine pancreatic trypsininhibitor. Bioorganicheskaya khimiya, 8(6), 776-816.

[12] Maksumov, I. S., Ismailova, L. I., \& Godjaev, N. M. 1983. The program for semiempirical calculation of conformations of the molecular complexes. J. Struc. Chem.(in Russian), 24, 147.

[13] Gaussian09, R. A. 2009. 1, Frisch MJ, Trucks GW, Schlegel HB, Scuseria GE, Robb MA, Cheeseman JR, Scalmani G., Barone V., Mennucci,B. Petersson GA et al., Gaussian. Inc., Wallingford CT.

[14] Becke, A. D. 1993. Density-functional thermochemistry. III. The role of exact exchange. The Journal of chemical physics, 98(7), 5648-5652.

[15] Funnell, N. P., Dawson, A., Francis, D., Lennie, A. R., Marshall, W. G., Moggach, S. A., Parsons, S. 2010. The effect of pressure on the crystal structure of L-alanine. CrystEngComm, 12(9), 2573-2583.

[16] Pawlukojć, A., Hołderna-Natkaniec, K., Bator, G., Natkaniec, I. 2014. L-glutamine: Dynamical properties investigation by means of INS, IR, RAMAN, $1 \mathrm{H}$ NMR and DFT techniques. Chemical Physics, 443, 17-25. 